\title{
Synthesis of Fast and Collision-free Folding of Polyhedral Nets
}

\author{
Yue Hao \\ George Mason University \\ Fairfax, VA \\ yhao3@gmu.edu
}

\author{
Yun-hyeong Kim \\ Seoul National University \\ Seoul, South Korea \\ yunhyk1202@gmail.com
}

\author{
Jyh-Ming Lien \\ George Mason University \\ Fairfax, VA \\ jmlien@cs.gmu.edu
}

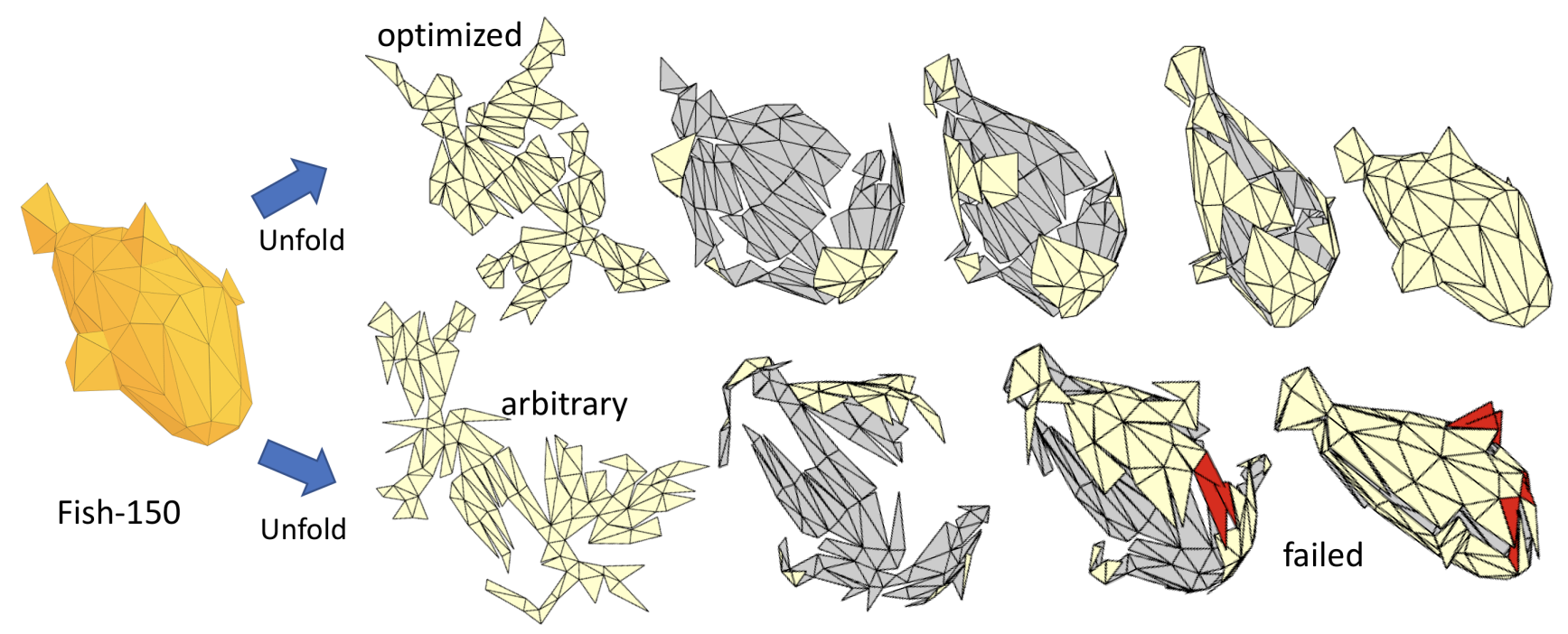

Figure 1: An optimized unfolding (top) created using our method and an arbitrary unfolding (bottom) for the fish mesh with 150 triangles (left). Each row shows the folding sequence by linearly interpolating the initial and target configurations. Selfintersecting faces, shown in red at bottom, result in failed folding. Additional results, foldable nets produced by the proposed method and an accompanied video are available on http://masc.cs.gmu.edu/wiki/LinearlyFoldableNets.

\begin{abstract}
A predominant issue in the design and fabrication of highly nonconvex polyhedral structures through self-folding, has been the collision of surfaces due to inadequate controls and the computational complexity of folding-path planning. We propose a method that creates linearly foldable polyhedral nets, a kind of unfoldings with linear collision-free folding paths. We combine the topological and geometric features of polyhedral nets into a hypothesis fitness function for a genetic-based unfolder and use it to map the polyhedral nets into a low dimensional space. An efficient learning strategy is used to optimize the fitness function to produce the optimal nets. We experimentally demonstrate that the proposed method can find linearly foldable nets for highly non-convex polyhedra with substantial complexity. The technique presented in the
\end{abstract}

Permission to make digital or hard copies of all or part of this work for personal or classroom use is granted without fee provided that copies are not made or distributed for profit or commercial advantage and that copies bear this notice and the full citation on the first page. Copyrights for components of this work owned by others than ACM must be honored. Abstracting with credit is permitted. To copy otherwise, or republish, to post on servers or to redistribute to lists, requires prior specific permission and/or a fee. Request permissions from permissions@acm.org.

SCF '18, fune 17-19, 2018, Cambridge, MA, USA

(C) 2018 Association for Computing Machinery.

ACM ISBN 978-1-4503-5854-5/18/06 . \$ \$15.00

https://doi.org/10.1145/3213512.3213517 paper will provide a powerful tool to enable designers, materials engineers, roboticists, to name just a few, to make physically conceivable structures through self-assembly by eliminating the common self-collision issue. It also simplifies the design of the control mechanisms when making deployable shape morphing devices. Additionally, our approach makes foldable papercraft more accessible to younger children and provides chances to enrich their education experiences.

\section{CCS CONCEPTS}

- Theory of computation $\rightarrow$ Computational geometry; Random search heuristics; • Computing methodologies $\rightarrow$ Mesh mod$e l s$;

\section{KEYWORDS}

Polyhedral nets, foldability analysis, optimization, motion planning, papercraft, self-folding robot, education

\section{ACM Reference Format:}

Yue Hao, Yun-hyeong Kim, and Jyh-Ming Lien. 2018. Synthesis of Fast and Collision-free Folding of Polyhedral Nets. In SCF '18: Symposium on Computational Fabrication, June 17-19, 2018, Cambridge, MA, USA. ACM, New York, NY, USA, 10 pages. https://doi.org/10.1145/3213512.3213517 


\section{INTRODUCTION}

For centuries, it is known that generating unfoldings from a 3D polyhedron, called polyhedral net [Dürer 1977], is a difficult problem [O'Rourke 2008]. Finding a net that can be folded rigidly along the crease lines from the unfolded 2D state to the target 3D state, while avoiding self-intersections, is more challenging even for simple shapes. For example, in a recently research led by An et al. [2014], self-folded 3D surfaces by uniform heating, researchers encountered the issue of collisions in some of their designs, which need a lot of further optimization.

\subsection{Not All Nets are Created Equal}

It is due to these difficulties, current practices treat net design and folding motion as two independent steps. However, there are numerous examples showing that the foldability of the nets created from a single polyhedron can vary significantly. For example, Fig. 1 shows two nets of a fish model. From the shapes of these nets, it is not possible to identify the better solution with respect to avoiding self-collisions until the model is simulated for folding. These nets also have substantial variations in computing folding paths. For example, in Fig. 1, it is about 350 times slower to finding a collisionfree folding motion for an arbitrary net than the optimized net.

\subsection{Challenges}

Recently, researchers have made progress in both generating valid nets and planning continuous foldings. However, given a polyhedron mesh, it remains computationally infeasible to enumerate all of its unfoldings and evaluate their foldability [Prautzsch and Straub 2005]. Existing methods either generate nets without considering foldability [Prautzsch and Straub 2005; Schlickenrieder 1997; Takahashi et al. 2011; Xi et al. 2016], plan motion on given nets ignoring the fact that net structures have significant impact on foldability [Demaine et al. 2004; Song and Amato 2004; Tachi 2009; $\mathrm{Xi}$ and Lien 2015], or require convexity to guarantee unfoldability and foldability [Kim et al. 2017]. The challenge of considering both net design and foldability remains significant for highly non-convex structures.

\subsection{Contributions}

This paper presents the first work that designs nets by optimizing its foldability as well as its quality of foldability. Our approach aims to create nets that are linearly foldable and uniformly foldable, if such nets exist. A net is linearly foldable if there exists a collisionfree straight line linearly interpolating the flat unfolded 2D state to the fully folded 3D state. Furthermore, a uniformly foldable net can fold under the constraint that their hinges must rotate at the same speed. To overcome high computational complexity in foldability analysis, we combine the topological and geometric features of polyhedral nets into a fitness function and use it to approximate the computationally expensive foldability metric. This synthesis of the domain knowledge with learning technique relieves us from the problem of the complex hypothesis model design and acquisition of large sample data that the traditional evolution control method faces.

We will demonstrate that the polyhedral nets generated using our method are, on average, up to 87 times faster for the planner to fold. For some non-convex shapes, our algorithm can converge to linearly foldable nets if such net exists, in which cases, the folding planning time is orders of magnitude faster than arbitrarily generated nets. We will also show that the linear foldable nets can facilitate papercraft fabrication in our user study experiment, and our technique provides low cost, easily foldable 3D models to younger children and offers chances to enrich their learning experiences

\section{BACKGROUND}

This section reviews recent progress in generating valid unfoldings, planning folding motion, and the concept of fitness approximation and evolution control.

The concept of self-folding machines has recently inspired many innovations [Davis et al. 2015; Felton et al. 2014; Liu et al. 2012]. Especially for robots made from low costs $2 \mathrm{D}$ active material that can be folded into 3D shape using light or heat [An et al. 2014; Shigemune et al. 2015]. However, most of these self-folding structures cannot perform complex folding motions, e.g., can only uniformly rotate their hinges [Sundaram et al. 2017]. Even for self-folding robots equipped with mechanical hinges [An et al. 2011; Felton et al. 2014], it is highly desirable to reduce the complexity of folding motion. These limitations and constraints raise a problem: given a target 3D shape, how can we design a 2D pattern that can be rigidly folded into the desired 3D shape without stretching or self-collision by following a folding motion that is as simple as possible? In the existing literature, this designing problem has only been addressed for convex shapes. Demaine et al. [2011] presented a polynomial time algorithm that can unfold and fold all convex polyhedra without self-intersection using source unfolding [Miller and Pak 2009].

In the rest of this paper, we use unfolding to denote the process of creating a net without considering motion and use folding exclusively to address the motion aspect of the problem.

\subsection{Polyhedra Unfolding}

Given a polyhedron $P$, the problem of polyhedra unfolding seeks a function $u(P)$ that transforms the faces of $P$ rigidly to produce a net $N$. In this work, we will focus on edge unfolding that cuts along the edges of the 3D meshes. Finding an edge unfolding of $P$ is to find a spanning tree in the dual graph of $P$. The difficulty is to ensure the flattened faces in a spanning tree do not overlap, in which case the spanning tree is a net. The unfolding problems are much easier if $P$ is convex. Heuristic methods [Prautzsch and Straub 2005; Schlickenrieder 1997] have been developed to assign weights to each dual edge such that the minimum spanning tree of the dual graph has a high probability of becoming a net.

Finding nets of non-convex shapes is a much more challenging task. Recently, genetic-based approaches [Takahashi et al. 2011; Xi et al. 2016] were proposed to evolve the unfoldings by mutating the cut edges until a net with zero overlaps is found. Their unfolding function $u(P, f)$ is parameterized by a fitness function $f: N \rightarrow \mathbb{R}$ that evaluates an unfolding $N$. An examples of $f(N)$ used in [Xi et al. 2016] is defined as:

$$
f(N)=-\left(\lambda_{0} \delta_{0}+\lambda_{1} \delta_{1}\right)
$$


where $\delta_{0}$ is the number of overlaps in the unfolding $N, \delta_{1}$ is the number of hyperbolic vertices that cause local overlaps in $N$, and $\lambda_{0}$ and $\lambda_{1}$ are user parameters. The value $f(N)$ of a net (i.e., an unfolding without overlaps) $N$ is 0 , however, it is not guaranteed that $N$ can be folded back to $P$ without self-intersection [Biedl et al. 2005].

\subsection{Folding Motion}

Planning folding motion is nontrivial. Early research focused on folding origami. Demaine et al. [2004] proved that a continuous folding motion always exists if the faces need not remain rigid. Tachi et al. [2009] proposed a heuristic method to continuously fold rigid origami via gradient descent. Origami usually have many faces but low Degrees of Freedom (DoF) due to the closure constraints. On the other hand, polyhedral nets have high DoF that is as large as the number of faces. The motion of these nets is typically found by a motion planner that models the net as a tree-like articulated robot. For example, Song and Amato [2004] proposed a PRM-based planner for nets generated from non-convex polyhedra. Recently, another motion planner has been developed by Xi and Lien [2015] that focuses on finding implicit folding order of a net (Fig. 2). Although these works provide effective algorithms to fold a given unfolding (net or origami), the structure of the unfoldings remains fixed in these approaches.
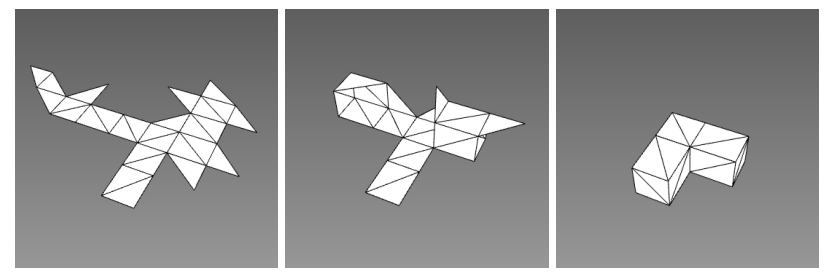

Figure 2: Folding a non-linearly foldable net requires an implicit ordering; the planner must uncover this order and take two folding steps in correct sequence to avoid selfintersections. The computational cost for such planning can be tremendous for large nets.

\subsection{Evolution-based Designs and Evolution Control}

Evolution algorithms are widely found in many applications involving morphology designs of linked structures. Lund et. al. [1997] use genetic algorithms to encode the body parameters of robot hardware into a morphological space. These parameters, e.g. robot wheel size, are then evolved through evaluating of the robot's performance on a specific task in the simulation. The idea of evolving virtual creatures, originally attempted by Sims [1994], where the robot morphology is represented in directed graphs. This method evolves new topologies but lacks improvements in complexity and does not scale to large parameter spaces. Some recent researches were able to evolve more complex robots using compositional pattern-producing networks [Auerbach and Bongard 2010, 2012; Cheney et al. 2013]. Even with the advanced encoding methods, evolving folding robots is still computationally infeasible with constraint kinematics of folding robots. Our work seeks to combine the domain knowledge of polyhedral nets to facilitate the encoding.

A naive unfolder $u\left(P, f^{*}\right)$ uses $f^{*}$ as a fitness function in the genetic-based algorithm. However, evaluating the foldability using motion planning is extremely time consuming; a single call of motion planner involves a large amount of sampling and collision checking and can take minutes to hours to complete. Additionally, as many planners are sampling-based algorithm, the estimation of motion planning time is stochastic. In order to obtain a stable estimation of foldability landscape, one can either sample the fitness function multiple times, or average over more individuals. Both methods will, again, dramatically increase the computational cost.

The fitness approximation methods [Jin 2005] have been widely applied to similar problems. Specifically, the functional approximation methods can be used in our problem domain. In functional approximation, an alternate and explicit expression is constructed for the fitness function. Take the most popular polynomial approximation for example, the constructed fitness function has the following form: $f^{\prime}=w_{0}+\sum_{i} w_{i} x_{i}+\sum_{i<j} w_{i+j} x_{i} x_{j}$, where $\left\{x_{i}\right\}$ is a set of criteria used to approximate $f^{*}$ and they are combined by coefficients $W=\left\{w_{i}\right\}$. The coefficients $W$ are then optimized using evolution control [Jin 2005] so that $\left|f^{\prime}-f^{*}\right|$ is minimized.

The success of fitness approximation methods stems from the sparse evaluation of the computationally expensive objective $f^{*}$; only once in each iteration of evolution control. The functional approximation method is essentially a supervised regression learner embedded in the evolution control framework. However, in polyhedron unfolding, it is difficult to find a good hypothesis fitness function $f^{\prime}$, even using sophisticated non-linear models, to substitute the folding path planning time $f^{*}$. Minimizing $\left|f^{\prime}-f^{*}\right|$ relies on a large number of samples through evaluation of $f^{*}$, which remains extremely time-consuming to obtain.

Fortunately, because our goal is finding the foldable nets, obtaining a perfect approximation that minimizes the error of $\left|f^{*}-f^{\prime}\right|$ is unnecessary. In this paper, we take a new approach that obtains the optimal net without finding a perfect fitness approximation. This idea is essential to the success of our method.

\section{FOLDABILITY OF POLYHEDRAL NETS}

If a polyhedral net $N$ is modeled as a tree-like articulated robot, the configuration space (C-space) $\mathbb{C}$ of $N$ is the set of valid configurations $c=\left\{\theta_{i}\right\}$, where $\theta_{i}$ is the folding angle of each hinge. The free configuration space (C-free) is a subset of $\mathbb{C}$ where $N$ has no self-intersection. For self-folding machines made of active materials, the folding motion is more constrained, as most hinges can only rotate monotonically or uniformly at the same speed. Due to a lack of coverage in the existing literature, we provide the following definitions to better describe the foldability of nets under these constraints. Note that dynamics are often not considered in the context of computational folding, and in our discussion the folding motion is considered quasi-static.

Continuously Foldable. Continuous foldable means a net $N$ can be folded back to $P$ without self-collision regardless the complexity of the folding motion. 
Monotonically Foldable. The monotonic foldability is a special case in continuous foldability, where the angular velocity must remain positive $\dot{\theta}_{i} \geqslant 0$ or negative $\dot{\theta}_{i} \leqslant 0$ throughout the folding, as many active material driven hinges cannot reverse the direction.

Linearly Foldable. A net is linearly foldable if there exists a straight line connecting initial and target states in C-free. Linearly foldable net has the shortest folding path in C-free, and the length of the path is

$$
L_{l}=\sqrt{\sum_{i=1}^{n} \theta_{i}^{2}}
$$

Physically, a linearly foldable net can be folded under the constraint that all hinges rotate at a speed proportional to the folding angle.

Uniformly Foldable. Distinct from linear folding, uniformly foldable nets can fold under the constraint that their hinges must rotate at the same speed. The linear and uniform foldability are both a special case of monotonic foldability. The length of uniformly folding path is

$$
L_{u}=\sqrt{n \theta_{1}^{2}}+\sum_{j=1}^{n-1} \sqrt{(n-j)\left(\theta_{j+1}-\theta_{j}\right)^{2}}
$$

where $\theta_{j} \leqslant \theta_{j+1}$ for $j=1,2 . . n$. If all $\theta_{j}$ are equal, then $L_{l}=L_{u}$ and the linear folding path and uniform path is identical for a given net.

Note that $L_{u}$ is upper bounded by $\sum_{i=1}^{n} \theta_{i}$, so $\sqrt{2}>\frac{L_{u}}{L_{l}} \geqslant 1$. This suggests that the uniformly folding path is longer than linear one by a constant, which suggests that the uniform foldability may be slightly harder to achieve.

To evaluate the foldability of a net, motion planning time can be a useful metric. Shorter planning time usually indicates an easier foldable net. Thus, we let $f^{*}(N)=T_{N, \Pi} / T_{N, A}$ be the foldability metric of a net $N$, where $T_{N, \Pi}$ is the time needed to check the validity of the path $\Pi$, and $T_{N, A}$ is the time needed for a planner $A$ to generate a collision-free path $\Pi$. If a net $N$ is continuously foldable, $0<f^{*}(N) \leq 1$. And, if $N$ is linear or uniform foldable, $f^{*}(N)$ is 1 . Our objective is to design an unfolder $u\left(P, f^{*}\right)$ that maps a polyhedron $P$ to a net $N$ with $f^{*}(N)$ as close to 1 as possible.

\section{OUR METHOD}

Inspired by the ideas of fitness approximation and evolution control [Jin 2005], we use learning strategy (shown in Fig. 5) to overcome two major obstacles in both net design and robot design: large number of design parameters and expensive evaluation function We will show that our approach is computationally efficient and requires significantly fewer evaluations in each iteration.

\subsection{Geometric and Topological Features of Nets}

To construct a good hypothesis fitness function, we observe that different unfoldings for a polyhedron may have distinct values in geometric and topological features. For example, Fig. 3 shows four distinct unfoldings of the spherical polygon which meshed by 320 triangles, and their corresponding feature values are listed in Table 1.

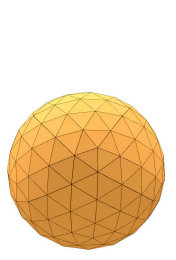

(a)

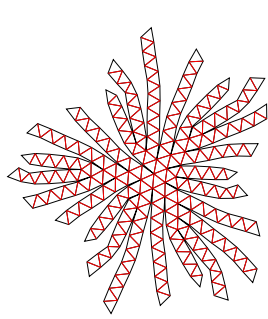

(b)

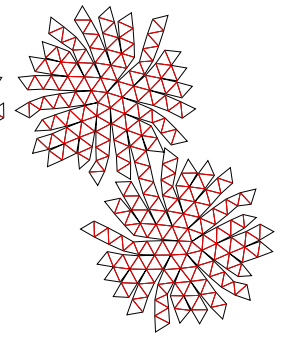

(c)

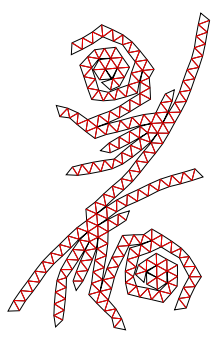

(d)

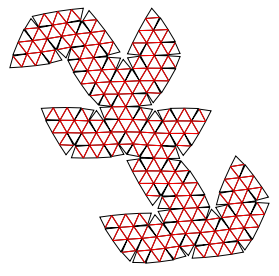

(e)
Figure 3: The spherical polyhedron model with 320 triangular faces (a) and some its representative nets (b)-(e).

Table 1: Geometric and topological features of the nets of a spherical polyhedron with 320 triangular faces shown in Fig. 3.

\begin{tabular}{|r|rrrr|}
\hline \multirow{2}{*}{ Characteristic } & \multicolumn{4}{|c|}{ Net } \\
\cline { 2 - 5 } & $(\mathrm{b})$ & (c) & $(\mathrm{d})$ & $(\mathrm{e})$ \\
\hline Avg. Branch Length & 11.83 & 5.36 & 11.27 & 1.94 \\
Avg. Geodesic Branch Length & 2.02 & 0.91 & 1.92 & 0.32 \\
Avg. Node Distance & 19.62 & 22.31 & 51.34 & 25.60 \\
Avg. Node Geodesic Distance & 3.39 & 3.85 & 8.84 & 4.51 \\
No. of Leaf Nodes & 24 & 44 & 18 & 104 \\
No. of One-Degree Nodes & 274 & 233 & 286 & 114 \\
No. of Two-Degree Nodes & 21 & 43 & 15 & 101 \\
Diameter & 40 & 45 & 124 & 66 \\
Cut Length & 48.26 & 47.87 & 47.92 & 45.62 \\
Hull Area & 23.17 & 13.41 & 33.59 & 6.02 \\
Border Cuts Length & 19.94 & 44.02 & 19.03 \\
Avg. Node Degree & 1.07 & 1.14 & 1.05 & 1.47 \\
Total Vertex Travel Distance & 103 & 120 & 246 & 119 \\
\hline
\end{tabular}

These features are insufficient to uniquely define a net, however, we presume that there are underlying correlations between these characteristics and shape of the nets. Our first goal is to map a polyhedral net $N$ into a feature space $\mathbb{X}$, in which the foldability can be evaluated for each point in $\mathbb{X}$. Three criteria are considered on selecting characteristics to construct our fitness function:

(1) There is a trade-off between the model complexity and training time. More characteristics generally lead to more robust model as the nets can be sparsely mapped into the feature space. However, it results in higher dimensional feature space and longer training time. 
(2) The selected features need to be efficiently computed, as they serve as a substitution for evaluating the foldability to save computing costs.

(3) In order to learn these features in a combined fitness function, the values must be normalized so they are within range $[0,1]$

Based on these considerations, the features in bold font type in Table 1 are chosen for constructing our fitness function and their definitions are summarized as follow.

Node Degrees. The first property is a topological feature defined as the ratio between the number of leaf (degree one) nodes and the total number of nodes in a net. Recall that nets are spanning trees, and each node has 1, 2 or 3 neighbors in triangular meshes. As we can observe in a net, the number of leaf nodes equals to the number of branches and approximates the branch lengths of the net.

Cut Length. Edges of a net are either cut or crease edges. Given a polyhedron with $|F|$ faces and $|E|$ edges, all net of this polyhedron must have $\sigma=2(|E|-|F|+1)$ cut edges and $|F|-1$ crease edges. The length of these $\sigma$ cut edges affects foldability and is usually minimized [Prautzsch and Straub 2005; Takahashi et al. 2011]. For example, cutting two longest edges of a narrow triangle creates a spike and these spikes are likely to collide. The cut length is upper bounded and lower bounded by the total length of $\sigma$ longest edges and $\sigma$ shortest edges, respectively, thus is normalized by these bounds.

Border Cuts Length. Cut length does not vary a lot if the polyhedron is tessellated with (nearly) equilateral triangles. Thus, we introduce the concept of border cuts to capture additional features. The intuition behind this is that some cut edges can be easily "zipped" without collision. On the contrary, pairs of matching border cuts are often separated far away and harder to be joined back. The border cut length is normalized by the total cut length.

Hull Area. The convex hull bounding the flattened net provides information about the clearance between branches. If the hull area is close to the total area of the net surface, this would indicate that the net is tightly bounded. We use the ratio of the surface area with respect to the hull area as a normalized factor in our fitness function.

\subsection{Enhanced Genetic-based Unfolder}

In the previous subsection, we mapped nets into a low dimensional feature space $\mathbb{X}$. We now discuss how to generate nets that corresponds to a particular region in $\mathbb{X}$. Recall that in Section 2.1, we reviewed the state-of-art genetic-based unfolder that generates valid unfoldings. We can enhance this genetic-based unfolder to generate unfoldings that satisfy desired topological and geometric features, using the following fitness function

$$
f^{\prime}= \begin{cases}f \text { in Eq. (1) } & \text { if net is invalid } \\ \beta+\hat{f}\left(x_{i}\right) & \text { otherwise }\end{cases}
$$

Firstly, the unfolder utilizes $f$ in Equation (1) to generate valid unfoldings without overlapping faces. Secondly, it uses $\hat{f}\left(x_{i}\right)$ to further evolve the nets, where $x_{i}$ denotes the quantified features in $\mathbb{X}$. The constant $\beta$ is used to ensure $\beta+\hat{f}\left(x_{i}\right)>0 \geq f$.

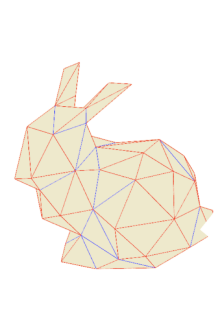

(a)

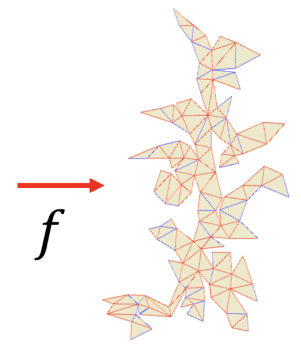

(b)

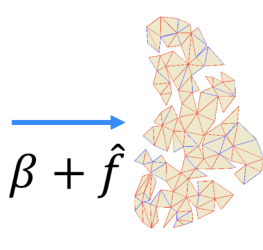

(c)
Figure 4: An example of the enhanced genetic-based unfolder that minimizes the Hall Area feature. (a) The original 3D model. (b) A valid unfolding with Hall Area $=31.6$ (c) A further evolved unfolding with Hall Area $=19.2$.

Shown in Fig. 4, the enhanced genetic-based unfolder can minimize the hull area of the unfolding for the bunny model by setting $\hat{f}$ negatively correlated to the Hull Area feature. Similarly, we can construct a hypothesis fitness that combines all the selected features that can potentially evolve the nets towards the maximum foldability. Due to the page limits, in this paper, we focus on the design of the learning framework. Readers interested the design of the hypothesis fitness functions should refer to a companion paper [Hao et al. 2018] for the full technical details.

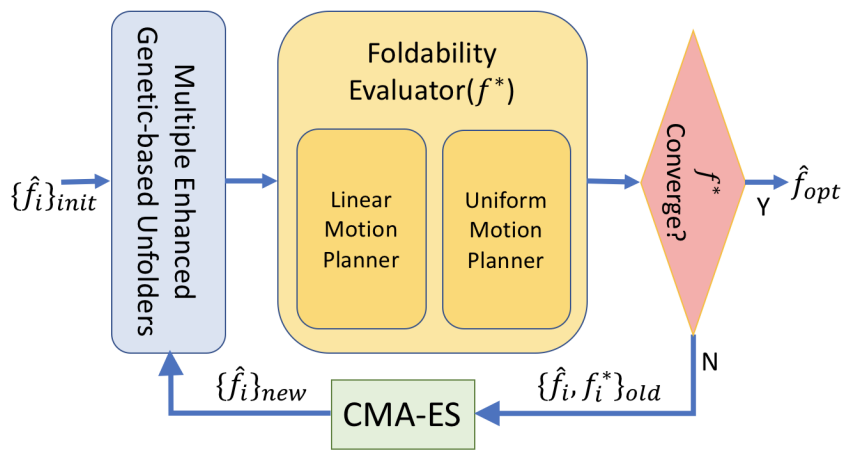

Figure 5: The framework of our learning strategy. In each iteration, multiple enhanced genetic-based unfolders run in parallel to generate polyhedral nets using the assigned fitness functions $\left\{\hat{f}_{i}\right\}$. The best individuals are then evaluated by the foldability evaluator which is either a linear or an uniform motion planner. An external CMA-ES optimizer then updates the fitness functions $\left\{\hat{f}_{i}\right\}$ according to their performance $\left\{f_{i}^{*}\right\}$, and generates a new set of fitnesses. This process is repeated until the evaluation converges.

\subsection{Optimizing the Fitness Function}

Recall that in Section 2.3, the naive unfolder $u\left(P, f^{*}\right)$ is infeasible because the fodability evaluator $f^{*}$ is computationally expensive. In practice, the genetic-based unfolder typically uses a population of 400 unfoldings, and takes about 300 evolutions in the enhancement 

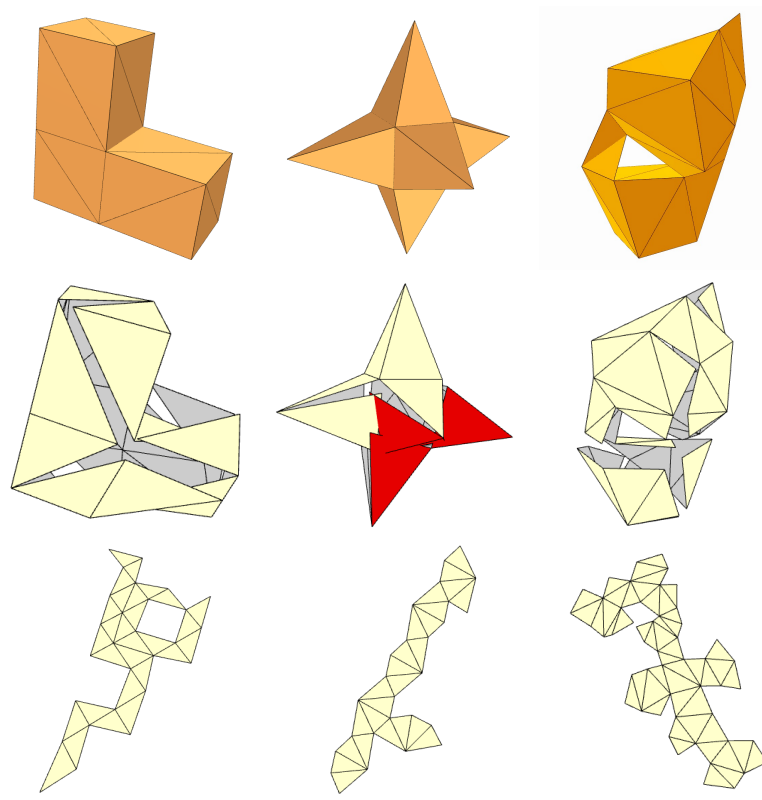

(a) $\mathrm{L}-28$

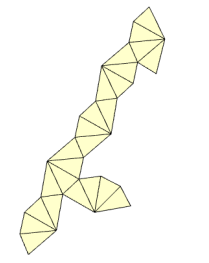

(b) Star-24

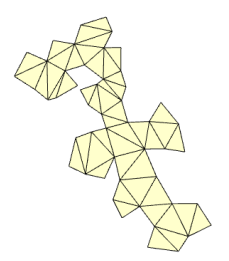

(c) Moneybox-48
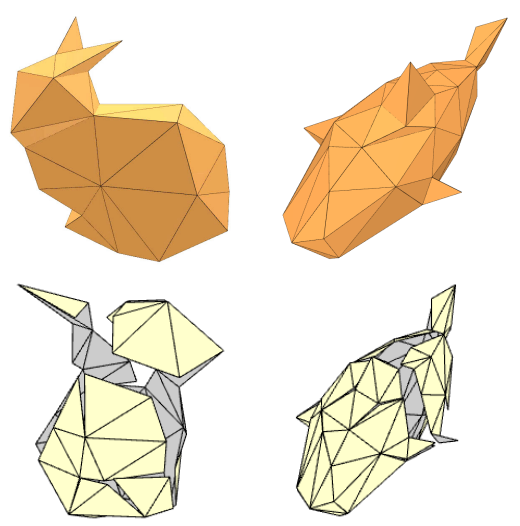

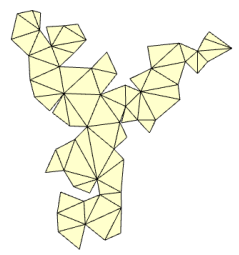

(d) Bunny-64

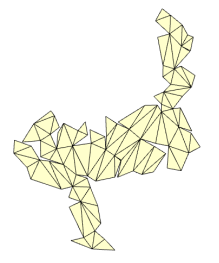

(e) Fish-96

Figure 6: First row: the models used in our experiments. Second row: the intermediate configuration on the linear folding path of the nets in the third row. The star shape has no linearly foldable net. Third row: one of the most continuously foldable nets for the models in the first row, generated using our proposed method.

step. In this case, calling $f^{*}$ for 120,000 times is extremely timeconsuming even for simple nets. Similarly, the traditional evolution control and function approximation method require even more computation to fully approximate $f^{*}$ with $f^{\prime}$ using the regression learner. Therefore, we propose a new learning strategy as shown in Fig. 5.

This learning strategy is a sampling based optimizer. In every iteration, we use a set of enhanced genetic-based unfolder $\left\{u\left(P, \hat{f}_{i}\right)\right\}$, each is assigned with a slightly different $\hat{f}_{i}$. Unlike the evolution control method, we do not evaluate all the individuals in the final generation of the evolution. Instead, we only evaluate the best individual (the individual with highest value of fitness) produced by each unfolder. In experiments, we typically run 40 unfolders in parallel, and the linear foldable nets often emerge within 20 iterations for simple models. Thus, we only need 800 calls of the evaluator. Due to the non-convexity of $f^{*}$, we use the Covariance Matrix Adaptation Evolution Strategy (CMA-ES) algorithm [Hansen et al. 2003] as an external optimizer to update the fitnesses. The initial guess $\left\{\hat{f}_{i}\right\}_{\text {init }}$ is typically generated at random. However, we can reuse the $\hat{f}_{\text {opt }}$ obtained from previous training on simple models to accelerate the training of complex 3D models. This iterative relaxation technique is discussion in the experiment section.

\section{EXPERIMENTS AND RESULTS}

In this section, we evaluate foldability of our optimized polyhedral nets in terms of path planning time (Sections 5.1). To further increase training efficiency of the proposed learning framework, we will discuss an optional optimization step in Section 5.2. We then demonstrate the uniformly folding with simulated hinge controller in Bullet Physics environment in Section 5.3. Finally, a user study is conducted to show the linearly foldable nets are easier for human to fold (Section 5.4).

\subsection{Optimized Polyhedral Nets}

We first show that the optimized unfolder generates more foldable polyhedral nets than arbitrary nets produced by an existing unfolder discussed in Section 2.2. We evaluate the foldability using the Average Path Planning Time APPT. The metric APPT is defined as follow: (1) For a polyhedron, we unfold it into 40 nets; (2) For each net, we plan the folding path 10 times using a given motion planner; (3) APPT is the average planning time for the 400 paths (40 nets $\times 10$ paths/net).

All experimental results reported in this section are set up as follows. To learn the fitness function, we run 40 genetic-based unfolders and the maximum training iteration is 50 . The population size within the genetic-based unfolder is 400 , and the maximum number of generations is 200 for polyhedra with less than 50 faces and 300 for over 50 faces. In each iteration, 40 best individuals are produced, and we use motion planner to evaluate each net 10 times and report the average. Parallelization is used in most stages. All data are collected from a $\mathrm{C}++$ implementation on a workstation with two $2.3 \mathrm{GHz}$ Intel CPUs.

The 3D models in Figs. 6 are used in our experiment. Among these models, only the star is not linearly foldable. Our algorithm uncovers the linearly foldable nets if such nets exist. Table 2 clearly shows that the optimized nets have much smaller APPTs, thus are easier to fold. 


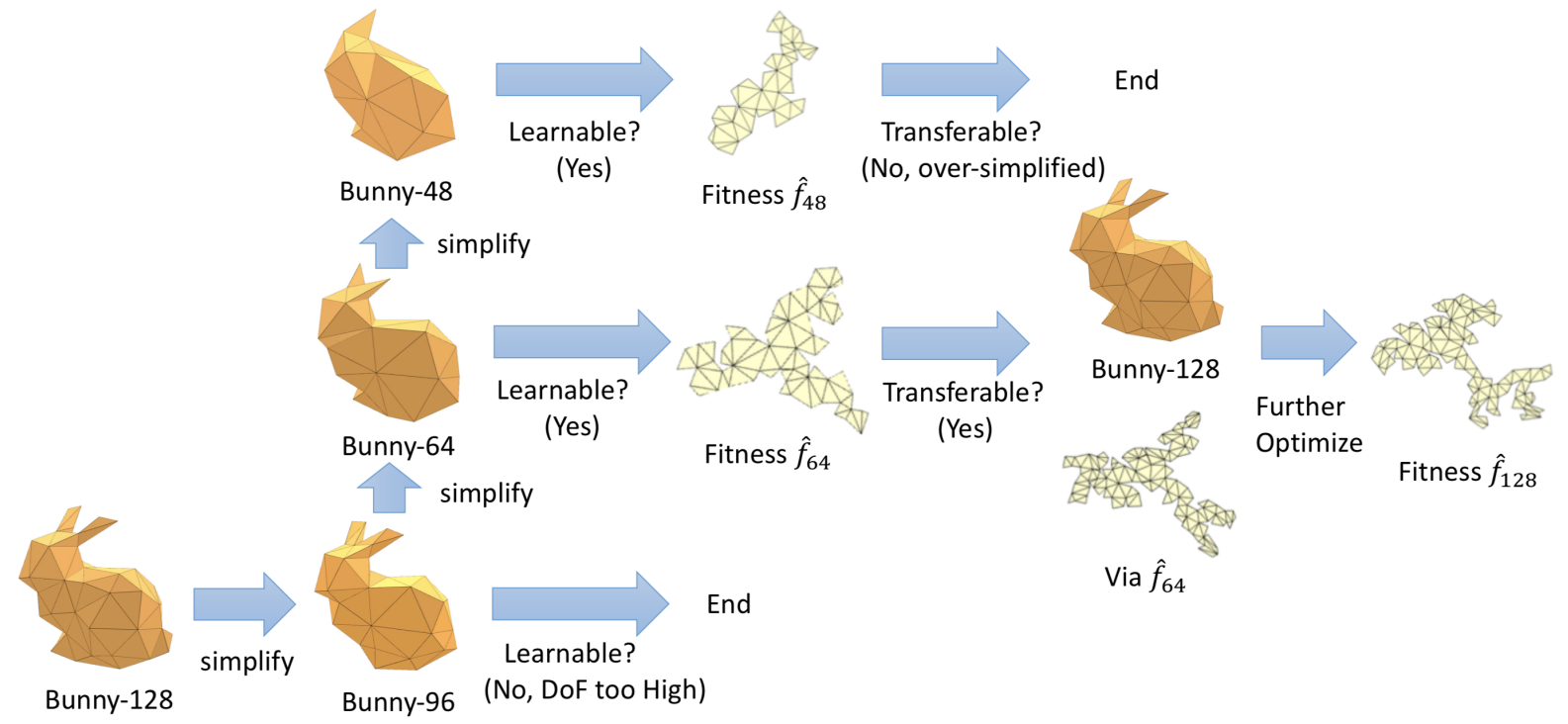

Figure 7: An optimal fitness $\hat{f}_{128}$ for Bunny-128 is obtained from $\hat{f}_{64}$ trained on Bunny-64, which is used as initial fitness for the learning of $\hat{f}_{128}$. If we only simplify it to Bunny-96, it is still a relatively complex model. However, if we reduce it to Bunny-48, it results in loss of details and non-convexity of the $3 \mathrm{D}$ model (e.g. one of the ears of the bunny is lost), the learning converges to a local optimal and $\hat{f}_{48}$ cannot be transferred to the original model.

Table 2: Comparison of APPT of Arbitrarily Generated Nets and Nets Generated Using Optimized Unfolder

\begin{tabular}{|c|c|r|r|r|}
\hline \multirow{2}{*}{ Model } & \multirow{2}{*}{ DoF } & \multicolumn{2}{|c|}{ APPT (s) } & \multirow{2}{*}{ Speedup } \\
\cline { 3 - 4 } & & Arbitrary & Optimized & \\
\hline L-28 & 27 & 0.892 & 0.021 & $42.5 \mathrm{x}$ \\
Star-24 & 23 & 17.587 & 1.195 & $14.7 \mathrm{x}$ \\
Moneybox-48 & 47 & 59.546 & 1.211 & $48.2 \mathrm{x}$ \\
Bunny-64 & 63 & 100.223 & 1.152 & $87.0 \mathrm{x}$ \\
Fish-96 & 95 & 82.418 & 2.923 & $28.1 \mathrm{x}$ \\
\hline
\end{tabular}

\subsection{Iterative Relaxation}

Conducting trainings on polyhedra with 100 faces or more remains time consuming. We employ transfer learning [Pan and Yang 2010] to reduce the complexity of the problem by remeshing the polyhedron with fewer faces. The simplified mesh, though may lose details, retain most geometric characteristics. Therefore, the fitness function trained from the simplified model is used as the initial fitness function to train the original model. Table 3 shows that the training time rises dramatically as the DoF increases, e.g., from 39 mins to 427 mins when the bunny increases from 48 faces to 128 faces. Table 3 also shows that transfer learning can significantly accelerate the training, e.g. only 76 mins comparing to $427 \mathrm{mins}$ for the bunny model. Fig. 7 illustrates the transfer learning of the bunny.

Fig. 8 shows results for more complex models obtained using the transfer learning strategy. Finding a linear folding path for these nets is almost instantaneous, while an arbitrarily generated net needs half an hour of path planning time.
Table 3: Comparison of Training Time (min.) for the first 20 Iterations on the Simplified Models with 48, 64, and 96 Faces, and the Original Model with or without Transfer Learning (TL).

\begin{tabular}{|c|c|c|c|c|c|c|}
\hline \multirow{2}{*}{$\begin{array}{c}\text { Simplified } \\
\text { Model }\end{array}$} & \multicolumn{3}{|c|}{ Num. of Faces } & \multirow{2}{*}{$\begin{array}{c}\text { Original } \\
\text { Model }\end{array}$} & \multirow{2}{*}{ w/o TL } & \multirow{2}{*}{$\mathrm{w} / \mathrm{TL}$} \\
\hline & 48 & 64 & 96 & & & \\
\hline Bunny & 39 & 47 & 152 & Bunny-128 & 427 & 76 \\
\hline Fish & 35 & 44 & 95 & Fish-150 & 521 & 102 \\
\hline Moneybox & 46 & 71 & 162 & Moneybox-128 & 502 & 77 \\
\hline
\end{tabular}

\subsection{Simulated Hinge Controller}

We also implement our polyhedral nets as hinge robots in Bullet Physics simulation to show that it is possible to design a realistic hinge controller for uniformly foldable nets. Shown in Fig. 9, we apply the same impulse to the hinges of the $\mathrm{L}$ shape, and each triangular face in the net is a rigid body with small but non-negligible thickness and mass. For this particular shape, the target joint angles are either $\frac{\pi}{2}$ for mountain folds, $-\frac{\pi}{2}$ for valley folds, or 0 for non-folding creases, making it both linearly and uniformly foldable. Shown in Fig 10, the motion is not entirely uniform, due to the variable inertia of linked faces of different lengths, i.e. it is more difficult for a joint to lift heavier linked faces. Although the equal speed condition is not fully satisfied, the optimized net still have large clearance between its linked faces and folds perfectly into the 3D shape under Bullet's a widely acknowledged collision checker. 


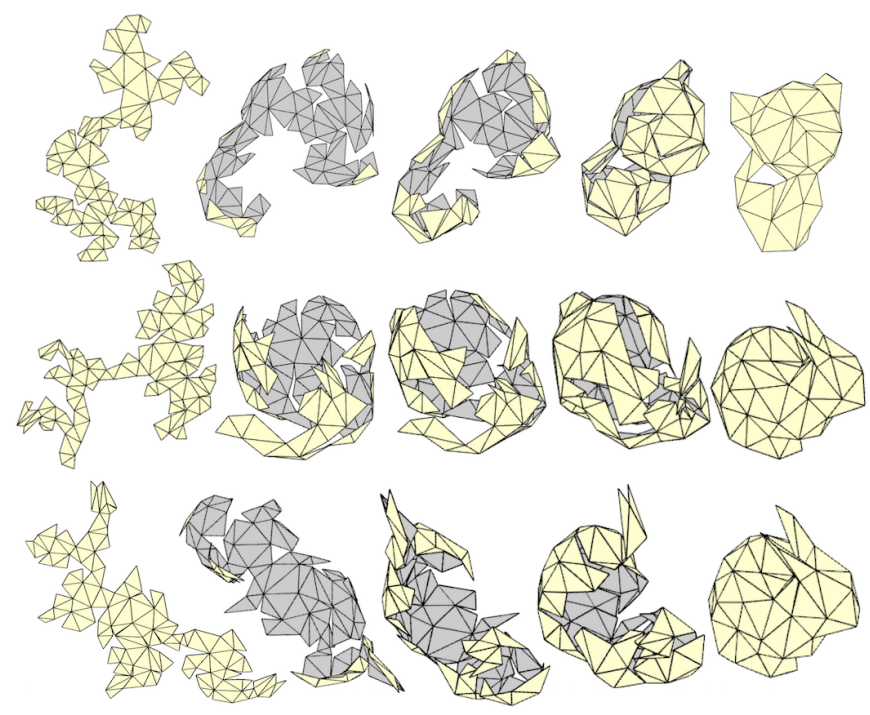

Figure 8: The folding sequences of optimal nets for complex models created using transfer learning. First row: linearly folding Moneybox-128; Second row: linearly folding Bunny128; Third row: uniformly folding Bunny-128.

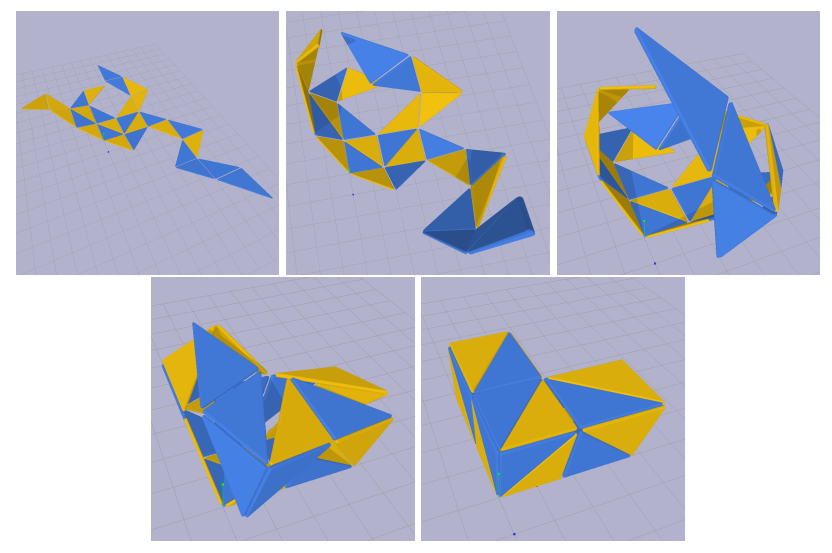

Figure 9: The uniformly foldable $L$ net folding in Bullet Physics simulation.

\subsection{User Study}

Polyhedral nets make great papercraft due to low cost and easy to manufacture and can be a great enjoyment for family members of all ages to fold. However folding complex nets can be frustrating due to a large amount of human work and difficulties, especially for children. Some earlier researches mainly focus on segmenting the nets [Xi et al. 2016] and divide the work for multiple people, while the approach may not produce desired artwork or causes sharing problems. One of the early motivation of this work is to find nets easy for a human to fold without reducing the complexity of the 3D mesh. We found the linearly foldable nets have three advantages over the arbitrarily generated nets in terms of folding: 1) the folding is relatively straightforward and does not involve ordering; 2 ) the

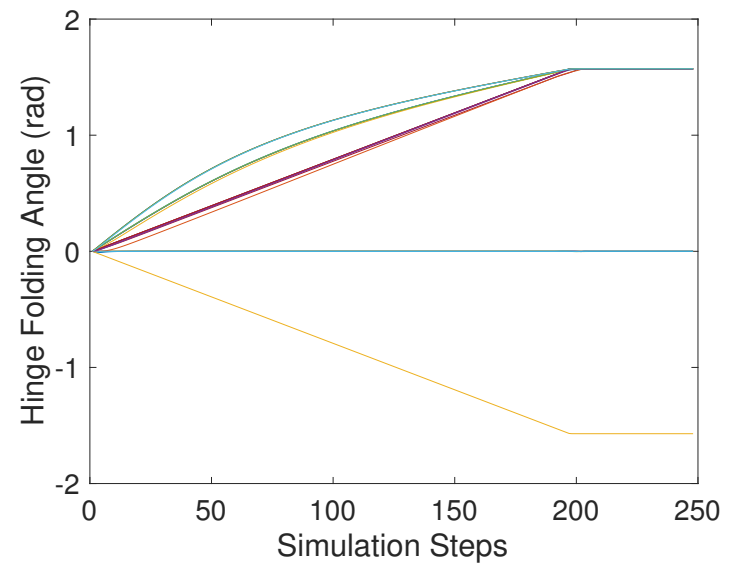

Figure 10: The hinge folding angles of uniformly foldable $L$ shape during simulation.

linearly foldable net tree has larger clearance between branches and subsequently less colliding faces; and 3) it is easy to make video instruction to facilitate human folding by showing the folding process generated by computer motion planner.

We carried out a user study experiment to physically validate our ideas in a classroom setting. The lesson focuses on the essential organs of the human body including a pair of lungs, a heart, a stomach, a pair of kidneys, a liver. Through an experience of putting together and making a 3-D puzzle by hand, this lesson can effectively provide students with a realistic and profound learning of their own bodily organs rather than the superficial perception, as well as increase students' interests of the organs. The lesson consists of a series of learning of the major bodily organs in order to recognize the importance of and understand the functions of them. In this lesson based on muscle memory, students will enhance their spatial sensibility and the activity fabricating 3-D organ puzzles will effectively help students develop their brain. Moreover, students will get to know that human body organs are parts of the body and work properly in specific areas of the body, and they will understand, if they do not keep their body in good shape and treat themselves right, that they will suffer from a crop of troubles such as illness. As a result, through this lesson, students will feel the actual size of their organs and understand there are the limitations of the human body, and subsequently, cultivate a good habit protecting their bodily organs.
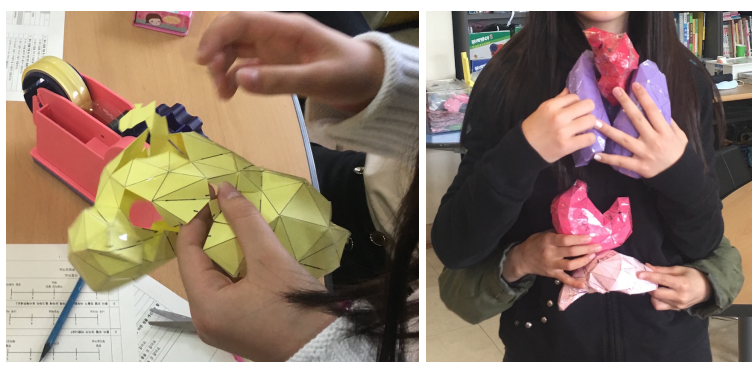

Figure 11: Our easily foldable nets used in biology classes. 

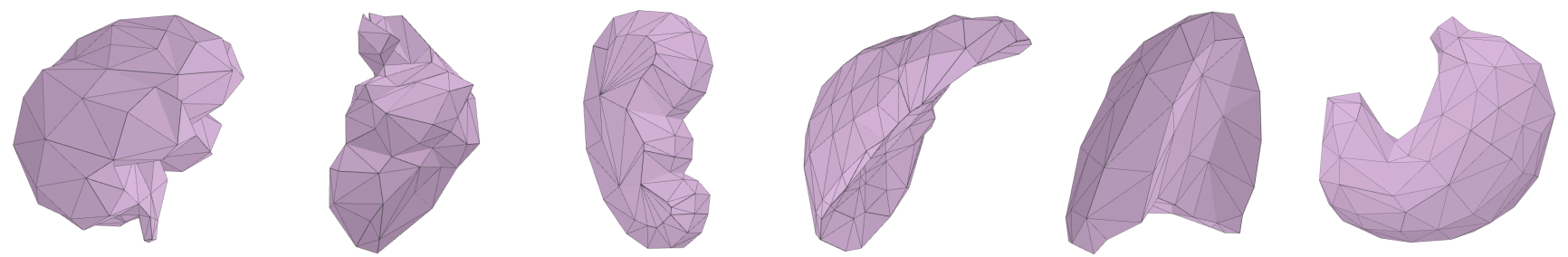

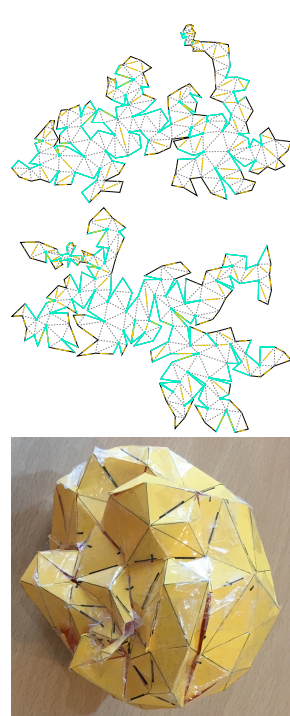

(a) Brain-210

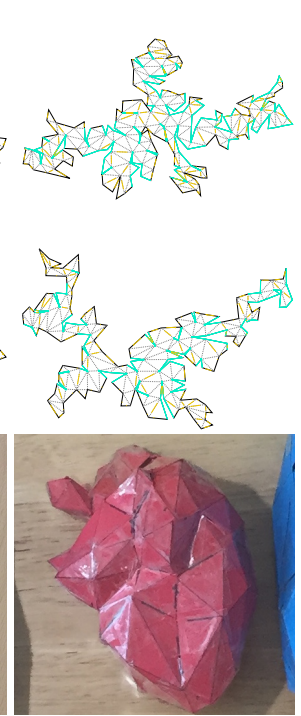

(b) Heart-215

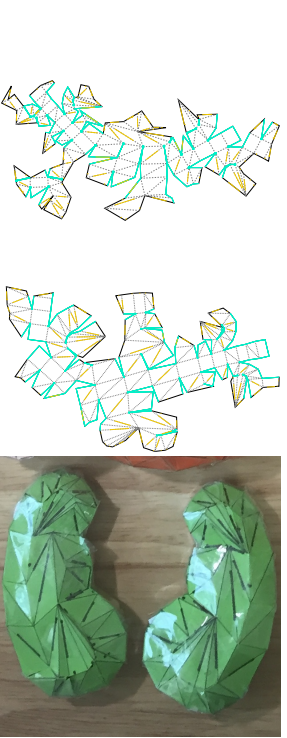

(c) Kiney-172
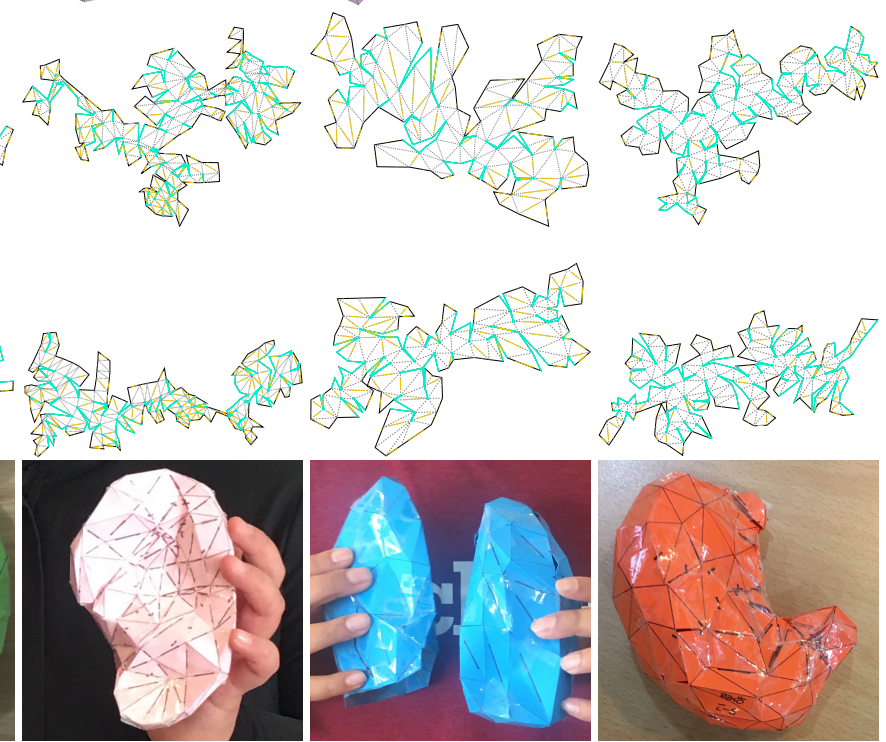

(d) Liver-274

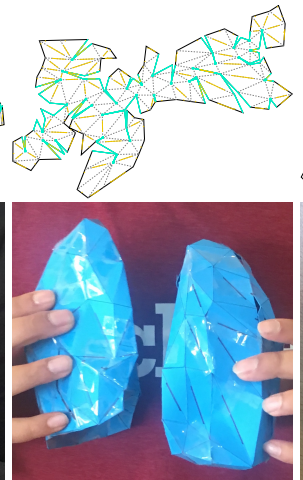

(e) Lung-144

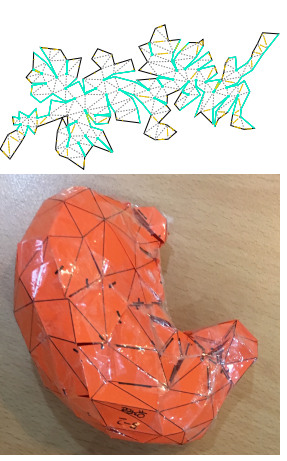

(f) Stomach-200

Figure 12: First row: the 3D models used in our human folding experiments; Second row: the arbitrary unfoldings of the first row; Third row: the linearly foldable nets for the models in the first row, generated using our proposed method; Forth row: handmade papercraft created by folding the nets.

We use the 3D shapes for six human organs: brain, heart, liver, a pair of lungs, a pair of kidneys and stomach, each of which has over 200 triangular faces. Both linearly foldable and arbitrary nets of these shapes are generated and a cutting plotter Cricut is used to cut these nets on thick paper. Then the nets are given to elementary school students in an age of around 11 years old. There are a total of 127 students in six classes, with a population of 20 to 22 in each class, participated in this experiment. Three classes (64 students) will only fold linearly foldable nets and the other three (63 students) will fold arbitrary nets. In each class, about five to six students will be in a group and each student chooses an organ and are given 50 to 60 minutes to finish. We compare the time they use to fold these nets and how far they are from complete within given time limit.

Table 4 shows the results of our experiment and Fig. 12 shows the models and nets the students folded. Due the limited class time, many students were not able to finish. We evaluate how far they are from finishing by visually inspect the percentage of completion by the end of the class, with 0 being the initially unfolded nets and 1 being the fully folded status. The collected data show that linearly foldable nets take a shorter time to fold in all cases except the heart.
Table 4: Human Folding Experiment Results, Avg. Folding Time Shown in Minutes, Avg. Completion Shown in Percentage, Lin $=$ Linearly Foldable Nets, Arb $=$ Arbitrary Nets

\begin{tabular}{r|c|c|c|c|c|c|c}
\hline \multirow{2}{*}{ Model } & \multirow{2}{*}{ Faces } & \multicolumn{2}{|c|}{ Avg. Folding Time } & \multicolumn{3}{c}{ Avg. Completion } \\
\cline { 3 - 8 } & & Arb & Lin & Diff & Arb & Lin & Diff \\
\hline Brain & 210 & 57.50 & 51.83 & 5.67 & 0.66 & 0.77 & 0.11 \\
Heart & 215 & 50.50 & 56.67 & -6.17 & 0.68 & 0.72 & 0.04 \\
Kidneys & $172 \times 2$ & 56.25 & 44.00 & 12.25 & 0.98 & 0.88 & -0.10 \\
Liver & 274 & 56.67 & 51.50 & 5.17 & 0.60 & 0.68 & 0.08 \\
Lungs & $144 \times 2$ & 51.60 & 47.22 & 4.13 & 0.84 & 0.96 & 0.13 \\
Stomach & 200 & 51.00 & 48.86 & 2.14 & 0.77 & 0.83 & 0.06 \\
\hline
\end{tabular}

\section{CONCLUSION AND DISSCUSSION}

In this paper, we proposed a computational design of fast and collision-free folding of polyhedral nets. Algorithmically creating a 2D crease pattern to realize the desired 3D structure involves two steps: (1) generating unfoldings (i.e., polyhedral net), and (2) creating collision-free folding motions that take polyhedral net back to the 3D shape. It is the current practice that net design and folding motion are decoupled and treated as two independent steps. This creates a major challenge in creating self-folding machines 
because, given a polyhedron $P$, the foldability of $P$ 's nets can vary significantly. Certain nets may not be foldable even using the most sophisticated folding motion planners.

Our research presents the seemingly counterintuitive results: there actually exist polyhedral nets with collision-free linearly path throughout the folding motion for those highly complex and highly non-convex geometries. The linearly and uniformly foldable nets open up new possibilities for manufacturing and fabrication that feature low-cost planar active material and self-folding into complex 3D structures. We also see great potential of the proposed work in educational fields. Similar to the pioneer research of HyperGami [Eisenberg and Nishioka 1997] in mathematical education, our work provides opportunities for students to learn abstract geometries in a tangible way.

For future work, we intend to use our algorithm in real-world folding-based structure fabrications, which may involve additional motion constraints. For example, in a recent work involving cutting and folding from planar metallic material using lasers [Lazarus and Smith 2017; Lazarus et al. 2018], the laser beam must not intersect with the unintended part of the folding shape during fabrication. This requires the net to satisfy a certain collision-free path other than linear or uniform folding, which raises new challenges in computationally generating such nets and paths.

\section{ACKNOWLEDGMENTS}

We thank Eun Sook Kim, a Master teacher at Seoul Hancheon Elementary School, for including our papercraft as part of the teaching materials in her classes and helping our experiments. Some early ideas in this papers were inspired from the discussions with In-Suk Choi of Seoul National University, Takahashi Shigeo of University of Aizu, Japan, Hsu-Chun Yen of National Taiwan University, and Zhonghua Xi of Google. We would also like to thank anonymous reviewers for their constructive comments. This work was supported in part by NSF EFRI-1240459 and CNS-1205260, AFOSR FA9550-17-1-0075 and Nvidia GPU Grant Program.

\section{REFERENCES}

Byoungkwon An, Nadia Benbernou, Erik D Demaine, and Daniela Rus. 2011. Planning to fold multiple objects from a single self-folding sheet. Robotica 29, 1 (2011), 87-102.

Byoungkwon An, Shuhei Miyashita, Michael T Tolley, Daniel M Aukes, Laura Meeker Erik D Demaine, Martin L Demaine, Robert J Wood, and Daniela Rus. 2014. An End-to-end Approach To Making Self-folded 3d Surface Shapes By Uniform Heating. In 2014 IEEE International Conference on Robotics and Automation (ICRA). IEEE, 1466-1473.

Joshua E Auerbach and Josh C Bongard. 2010. Dynamic resolution in the co-evolution of morphology and control. In Artificial Life XII: Proceedings of the Twelfth International Conference on the Synthesis and Simulation of Living Systems. MIT Press, 451-458.

Joshua E. Auerbach and Joshua C. Bongard. 2012. On the Relationship Between Environmental and Morphological Complexity in Evolved Robots. In Proceedings of the 14th Annual Conference on Genetic and Evolutionary Computation (GECCO '12). ACM, New York, NY, USA, 521-528. https://doi.org/10.1145/2330163.2330238

Therese Biedl, Anna Lubiw, and Julie Sun. 2005. When can a net fold to a polyhedron? Computational Geometry 31, 3 (2005), 207 - 218. https://doi.org/10.1016/j.comgeo. 2004.12.004 11th Canadian Conference on Computational Geometry.

Nick Cheney, Robert MacCurdy, Jeff Clune, and Hod Lipson. 2013. Unshackling Evolution: Evolving Soft Robots with Multiple Materials and a Powerful Generative Encoding. In Proceedings of the 15th Annual Conference on Genetic and Evolutionary Computation (GECCO '13). ACM, New York, NY, USA, 167-174. https://doi.org/10. 1145/2463372.2463404

Duncan Davis, Bin Chen, Michael D Dickey, and Jan Genzer. 2015. Self-folding of Thick Polymer Sheets Using Gradients of Heat. Fournal of Mechanisms and Robotics (2015).
Erik D. Demaine, Martin L. Demaine, Vi Hart, John Iacono, Stefan Langerman, and Joseph O'Rourke. 2011. Continuous Blooming Of Convex Polyhedra. Graphs and Combinatorics 27, 3 (2011), 363-376.

Erik D. Demaine, Satyan L. Devadoss, Joseph S. B. Mitchell, and Joseph O'Rourke. 2004. Continuous foldability of polygonal paper. In CCCG.

Albrecht Dürer. 1977. Underweyssung der Messung mit dem Zyrkel und Rychtscheyd. Nürnberg (1525). English translation with commentary by Walter L. Strauss The Painter's Manual, New York (1977).

Michael Eisenberg and Ann Nishioka. 1997. Polyhedral Sculpture: The Path from Computational Artifact to Real-world Mathematical Object. SIGCUE Outlook 25, 1-2 (Jan. 1997), 30-40. https://doi.org/10.1145/274375.274381

Samuel M. Felton, Michael Tolley, Erik D. Demaine, Daniela Rus, and Robert Wood. 2014. A Method For Building Self-folding Machines. Science 345, 6197 (2014), 644-646.

Nikolaus Hansen, Sibylle D Müller, and Petros Koumoutsakos. 2003. Reducing the time complexity of the derandomized evolution strategy with covariance matrix adaptation (CMA-ES). Evolutionary computation 11, 1 (2003), 1-18.

Yue Hao, Yun-Hyeong Kim, Zhonghua Xi, and Jyh-Ming Lien. 2018. Creating Foldable Polyhedral Nets Using Evolution Control. In Proceedings of the Robotics: Science and Systems Conference (RSS). Pittsburgh, Pennsylvania.

Yaochu Jin. 2005. A comprehensive survey of fitness approximation in evolutionary computation. Soft computing 9, 1 (2005), 3-12.

Yun-Hyeong Kim, Zhonghua Xi, and Jyh-Ming Lien. 2017. Disjoint Convex Shell and its Applications in Mesh Unfolding. In Symposium on Solid and Physical Modeling (SPM), also appears in fournal of Computer-Aided Design. Berkeley, California.

Nathan Lazarus and Gabriel L. Smith. 2017. Laser Forming for Complex 3D Folding. Advanced Materials Technologies 2, 10 (2017), 1700109. https://doi.org/10.1002/admt. 201700109 arXiv:https://onlinelibrary.wiley.com/doi/pdf/10.1002/admt.201700109

Nathan Lazarus, Adam Wilson, and Gabriel Smith. 2018. Contactless laser fabrication and propulsion of freely moving structures. 20 (04 2018).

Ying Liu, Julie K Boyles, Jan Genzer, and Michael D Dickey. 2012. Self-folding of polymer sheets using local light absorption. Soft Matter 8, 6 (2012), 1764-1769.

H. H. Lund, J. Hallam, and Wei-Po Lee. 1997. Evolving robot morphology, In Evolutionary Computation, 1997., IEEE International Conference on. Evolutionary Computation, 1997., IEEE International Conference on, 197-202. https://doi.org/10. 1109/ICEC.1997.592295

Ezra Miller and Igor Pak. 2009. Metric combinatorics of convex polyhedra: cut loci and nonoverlapping unfoldings. In Twentieth Anniversary Volume: Springer, 1-50. Joseph O'Rourke. 2008. Unfolding polyhedra.

Sinno Jialin Pan and Qiang Yang. 2010. A survey on transfer learning. IEEE Transactions on knowledge and data engineering 22, 10 (2010), 1345-1359.

Hartmut Prautzsch and Raphael Straub. 2005. Creating Optimized Cut-out Sheets for Paper Models from Meshes. In Proceedings of the 9th SIAM Conference on Geometric Design and Computing.

Wolfram Schlickenrieder. 1997. Nets of polyhedra. Master's thesis. Technische Universität Berlin.

Hiroki Shigemune, Shingo Maeda, Yusuke Hara, Uori Koike, and Shuji Hashimoto. 2015. Kirigami robot: Making paper robot using desktop cutting plotter and inkjet printer. In Intelligent Robots and Systems (IROS), 2015 IEEE/RSf International Conference on. IEEE, 1091-1096.

Karl Sims. 1994. Evolving Virtual Creatures. In Proceedings of the 21st Annual Conference on Computer Graphics and Interactive Techniques (SIGGRAPH '94). ACM, New York, NY, USA, 15-22. https://doi.org/10.1145/192161.192167

Guang Song and Nancy M Amato. 2004. A Motion-planning Approach To Folding: From Paper Craft To Protein Folding. Robotics and Automation, IEEE Transactions on 20, 1 (2004), 60-71.

Subramanian Sundaram, David S. Kim, Marc A. Baldo, Ryan C. Hayward, and Wojciech Matusik. 2017. 3D-Printed Self-Folding Electronics. ACS Applied Materials \& Interfaces 9, 37 (2017), 32290-32298. https://doi.org/10.1021/acsami.7b10443 arXiv:http://dx.doi.org/10.1021/acsami.7b10443 PMID: 28825288.

Tomohiro Tachi. 2009. Simulation of Rigid Origami. Origami 4 (2009), 175-187.

Shigeo Takahashi, Hsiang-Yun Wu, Seow Hui Saw, Chun-Cheng Lin, and Hsu-Chun Yen. 2011. Optimized topological surgery for unfolding $3 \mathrm{~d}$ meshes. In Computer Graphics Forum, Vol. 30. Wiley Online Library, 2077-2086.

Zhonghua Xi, Yun-Hyeong Kim, Young J. Kim, and Jyh-Ming Lien. 2016. Learning to Segment and Unfold Polyhedral Mesh from Failures. In Shape Modeling International (SMI); also appears in fournal of Computers \& Graphics. Berlin, Germany.

Zhonghua Xi and Jyh-Ming Lien. 2015. Continuous Unfolding of Polyhedra - a Motion Planning Approach. In 2015 IEEE/RSf International Conference on Intelligent Robots and Systems (IROS). Hamburg, Germany, 3249 - 3254. 